\title{
Meta-analysis of the influence of time interval between radical operation and preoperative chemoradiotherapy on complete pathological response in rectal cancer patients
}

\author{
Liangjie Zheng ${ }^{1}$, Weibo $\mathrm{Hu}^{1}$, Xue Zhen ${ }^{1}$, Yanxia Yang ${ }^{2 \star}$ \\ ${ }^{1}$ Department of Oncology, The People's Hospital of Leling City, ${ }^{2}$ Pharmacy of Traditional Chinese Medicine, Leling Hospital of \\ Traditional Chinese Medicine, Leling 253600, Shandong Province, China
}

${ }^{*}$ For correspondence: Email: yangyanxia1106@126.com

\begin{abstract}
Purpose: To evaluate the impact of interval between chemoradiotherapy (CRT) and surgery on rates of pathological complete response ( $p C R$ ).

Methods: A search was carried out from PubMed and Embase databases for literature related to clinical benefits in rectal cancer patients after surgery performed at different intervals following neoadjuvant therapy. The main endpoint was the rate of $p C R$. Relative risk (RR) of chance of a pCR among different intervals was assessed.

Results: Among 3462 screened individuals, 11 retrospective cohort studies representing 3462 relevant patients were qualified for inclusion in the study. The time intervals varied between $\leq 5$ weeks and $>12$ weeks. Thell studies were divided into 6 categories based on surgical timing. The potential association between the $p C R$ rate and time intervals of $5,6,7,8,10$ or 12 weeks was analyzed. Pooled $R R$ and 95 $\%$ confidence intervals $(\mathrm{Cls})$ of $\mathrm{pCR}$ rates at different intervals were plotted on a line chart. The highest plateau in the $R R$ of $p C R$ rates were noted in patients undergoing surgery beyond 7 weeks (RR, 1.60; $95 \% \mathrm{Cl}, 1.20-2.13 ; p=0.001)$ after the end of CRT.

Conclusion: Radical operation over 7 weeks following CRT results in the highest risk of $p C R$. The question as to whether this is associated with high long-term survival rate remains to be resolved.
\end{abstract}

Keywords: Rectal cancer, Time interval, Surgery, Chemotherapy, Radiotherapy

\begin{abstract}
This is an Open Access article that uses a funding model which does not charge readers or their institutions for access and distributed under the terms of the Creative Commons Attribution License (http://creativecommons.org/licenses/by/4.0) and the Budapest Open Access Initiative (http://www.budapestopenaccessinitiative.org/read), which permit unrestricted use, distribution, and reproduction in any medium, provided the original work is properly credited.
\end{abstract}

Tropical Journal of Pharmaceutical Research is indexed by Science Citation Index (SciSearch), Scopus, International Pharmaceutical Abstract, Chemical Abstracts, Embase, Index Copernicus, EBSCO, African Index Medicus, JournalSeek, Journal Citation Reports/Science Edition, Directory of Open Access Journals (DOAJ), African Journal Online, Bioline International, Open-J-Gate and Pharmacy Abstracts

\section{INTRODUCTION}

CRT after surgery has become a routine procedure of multimodal treatment in patients with locally-advanced rectal cancer $[1,2]$. A metaanalysis consisting of randomized-controlled studies suggested that preoperative CRT improves local control compared with surgery alone or surgery combined with neoadjuvant radiotherapy. Additionally, neo-adjuvant chemoradiotherapy may result in complete eradication of all viable tumor cells from the primary tumor site as well as from the regional lymph nodes, representing PCR in this setting [3-5]. Furthermore, complete response to preoperative CRT predicts better long-term outcomes with low 
rates of local recurrence and distant failure.

Currently, few small studies have investigated the influence of the length of the interval between CRT and surgery (CRT-surgery interval) on resectability, morbidity, and tumor response $[6,7]$. However, there is lack of level- 1 evidence that patients have obtained larger tumor shrinkage if the surgical timing was deferred for several weeks and conflicting data exist. The best time interval between CRT and surgery which yields the maximal tumor regression is still unknown, and quantitative summarization of the supporting evidence is not available. This systematic review aims to examine the impact of the CRT-surgery time interval on the rate of $\mathrm{pCR}$.

\section{METHODS}

\section{Data retrieval}

Two investigators searched PubMed and Embase databases for relevant article; electronic database searches were performed with the Boolean combination [(Interval or time or timing) and rectal and (carcinoma or cancer) and (Chemoradiotherapy OR Radiotherapy OR radiation OR neoadjuvant)] in all fields. In addition, the selected studies and related publications were screened for additional trials.

\section{Study selection}

The primary objective of this study was to evaluate the influence of time interval between CRT and surgery on pCR rate. We selected only those trials that directly compared the pCR rates among patients undergoing rectal cancer surgery at varying time intervals after CRT. Inclusion criteria: studies had to report comparable data on pCR rates after CRT. Studies that were not published as full reports, such as letters to editors and conference abstract should be eliminated. In case of multiple publications on the same study, the most recent information was used.

\section{Study assessment}

To avert the risk of bias during the process of data abstraction, two authors were responsible for data extraction. If the discrepancies occurred between two authors, they should be solved by agreement. Details about the author, number of patients, publication year, definition of the pCR, treatment information, patient baseline characteristics and pCR rates of different groups from the included studies were included. We collected data from each group separately when studies compared pCR rates among more than 2 time intervals.

\section{Statistical analysis}

Statistical analysis was performed by using the Stata 12.0 statistical software (Stata Corp, Texas, U.S.). To calculate RR, patients of longer interval group were compared only with those of shorter interval in the same clinical trial. RR together with the $95 \%$ confidence interval (Cl) was used as summary statistics for dichotomous data. The relationship between $\mathrm{pCR}$ rates and time intervals was analyzed by dividing patients into six categories based on the time interval between CRT and surgery. Statistical heterogeneity with 12 statistics was evaluated. $P$ $<0.05$ was considered statistical significance.

\section{RESULTS}

We researched a total of 2682 relevant clinical studies, of which 1887 were among other themes. In total, 30 articles were retrieved for full-text review. Of these, 8 articles were excluded because of lack of relevant data, 1 because there was no control arm, 7 because no mention was made of the precise cutoff point of CRT-surgery interval, and 5 because it did not report the postoperative pathologic outcome. The remaining 11 studies comprising of a total of 3462 individuals constituted the material for the current review. Review articles $(n=258)$, comments $(n=18)$, case reports $(n=121)$, letters $(n=17)$ and articles written in other languages $(\mathrm{n}=321)$ were excluded from subsequent analysis (Figure 1).

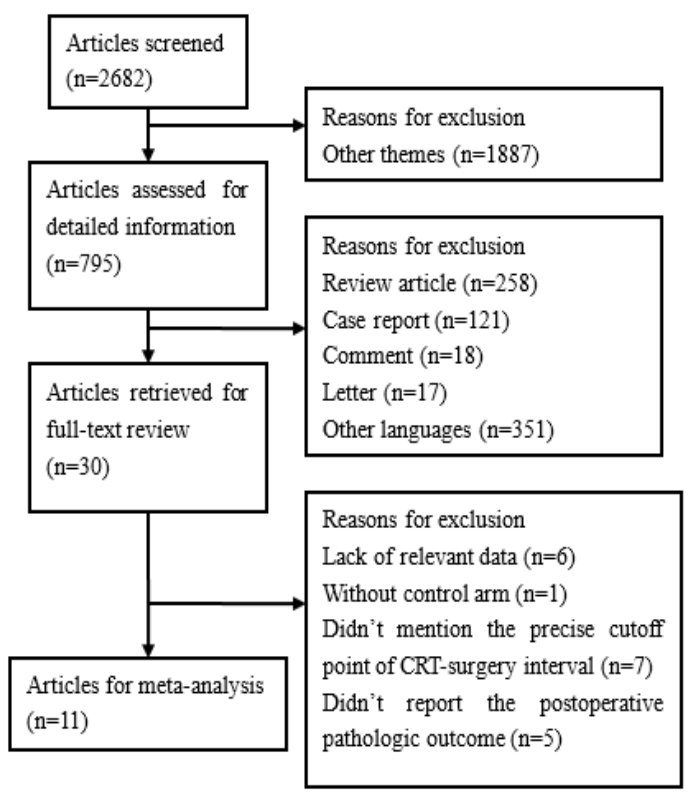

Figure 1: Flow diagram of the research selection 


\section{Study characteristics}

Eleven retrospective cohort studies were included and these studies were different in the time interval between CRT and surgery, ranged from $\leq 4$ weeks vs $>4$ weeks to $\leq 11$ weeks vs $>$ 11 weeks. All studies reported on patients who received CRT. Although preoperative radiotherapy was heterogeneous among included studies, most included studies administered 46 to 54 Gy delivered over 5-6 weeks. All of the studies were on patients who received 5-fluorouracil (FU) - based chemotherapy, though the chemotherapy regimens used were heterogeneous. The common factors influencing CRT - surgery intervals reported by most studies were the surgeons' policy regarding the timing of operation, the bed availability on the surgical ward and comorbidities. Complete pathological response was differently defined in the selected studies.

The mean tumor height to the anal verge was identified between the shorter and longer time intervals in each of the included studies. In most studies, neoadjuvant therapy was delivered to the patients diagnosed with stage II-III rectal carcinoma, or with tumors that threatened circumferential resection margin. None of the included studies reported a significant difference in distribution of clinical $\mathrm{T}$ - and $\mathrm{N}$ - stage in respect of shorter and longer time intervals trials.

\section{Methodological quality}

Eleven included studies were evaluated by two independent authors with a 6-star level of methodological quality based upon the Newcastle-Ottawa quality assessment scale.

\section{Complete pathological response}

An increasing pCR rate after neoadjuvant therapy was observed when patients were operated at an interval $>7$ weeks and a waiting interval of $\geq 8$ weeks was associated with a higher $\mathrm{pCR}$ rate. The highest $\mathrm{pCR}$ rates were noted in those undergoing surgery on 10 to 11 weeks following CRT in the large series [8]. Five studies reported a non-significant trend toward increased $\mathrm{pCR}$ rates in long interval group. No increase was noted in the pCR rate in 2 studies.

\section{pCR rate}

A total of 3462 patients from 11 studies were enrolled for subsequent analysis. Among patients treated with CRT, the summary rate of $\mathrm{pCR}$ was
15.8\% (95 \% Cl, $12.8 \%-18.8 \%)$, using a random-effects model.

\section{Subgroup analyses}

To investigate the specific contribution of time intervals of CRT-surgery to the occurrence of pCR, a meta-analysis was conducted to calculate the $R R$ related with time intervals at $5,6,7,8,10$ or 12 weeks when compared to controls (Figure 2). No heterogeneity was detected between different selected studies included in this metaanalysis. Using the fixed effect model, the pCR rate was considerably increased in patients undergoing surgery at 7-8 weeks (RR, 1.60; 95\% $\mathrm{Cl}, 1.20-2.13 ; P=0.01 ; \mathrm{RR}, 1.47 ; 95 \% \mathrm{Cl}, 1.22$ $-1.83 ; P=0.05)$. The $\mathrm{pCR}$ rate did not significantly differ between cohorts with shorter and longer time intervals in remaining cutoff points of $5,6,10$ or 12 weeks (all $P>0.05$ ). Sensitivity analyses excluding the data to control for patient with near pCR did not alter the results substantially (RR, 1.339; $95 \%$ Cl 1.145 to 2.056; $P=0.022)$.

We then plotted the pooled $\mathrm{RR}$ and $95 \% \mathrm{Cl}$ of pCR rates of different intervals in a line chart. The highest summary point estimate in the RR of pCR rates were observed in patients operated beyond 7 weeks after the end of CRT, which was associated with an approximate $60 \%$ higher chance of achieving pCR than operated before 7 weeks (RR, 1.60; $95 \% \mathrm{Cl}, 1.21-2.12 ; P=$ $0.002)$. The corresponding figures for $\geq 8$ weeks cutoff point were $47 \%(\mathrm{RR}, 1.45 ; 95 \% \mathrm{Cl}, 1.11$ $-1.83 ; P=0.025)$ (Figure 3).

\section{DISCUSSION}

Radiation-induced necrosis is dependent upon surgical timing. The impact of chemo-radiation therapy depends upon cell cycle. In addition, multiple cycles over several months may be required to observe the actual effect. The exact surgical timing after neo-adjuvant treatment for patients with rectal cancer still remains elusive. Many scholars attempts to identify an optimal surgical timing following preoperative radiation therapy start from the Lyon R90-01 investigation which examined and analyzed the time interval to surgery, (short and long intervals) following preoperative radiotherapy were analyzed [9,10]. Hence, there is enthusiasm for prolonging the currently accepted interval of 6-8 weeks in order to maximize the down-staging effect of CRT and increase the pCR rate. But will a longer interval even result in a superior rate of pCR? Is there an optimal interval for surgical intervention following neo-adjuvant chemoradiotherapy? The longer interval was associated with a higher percentage 


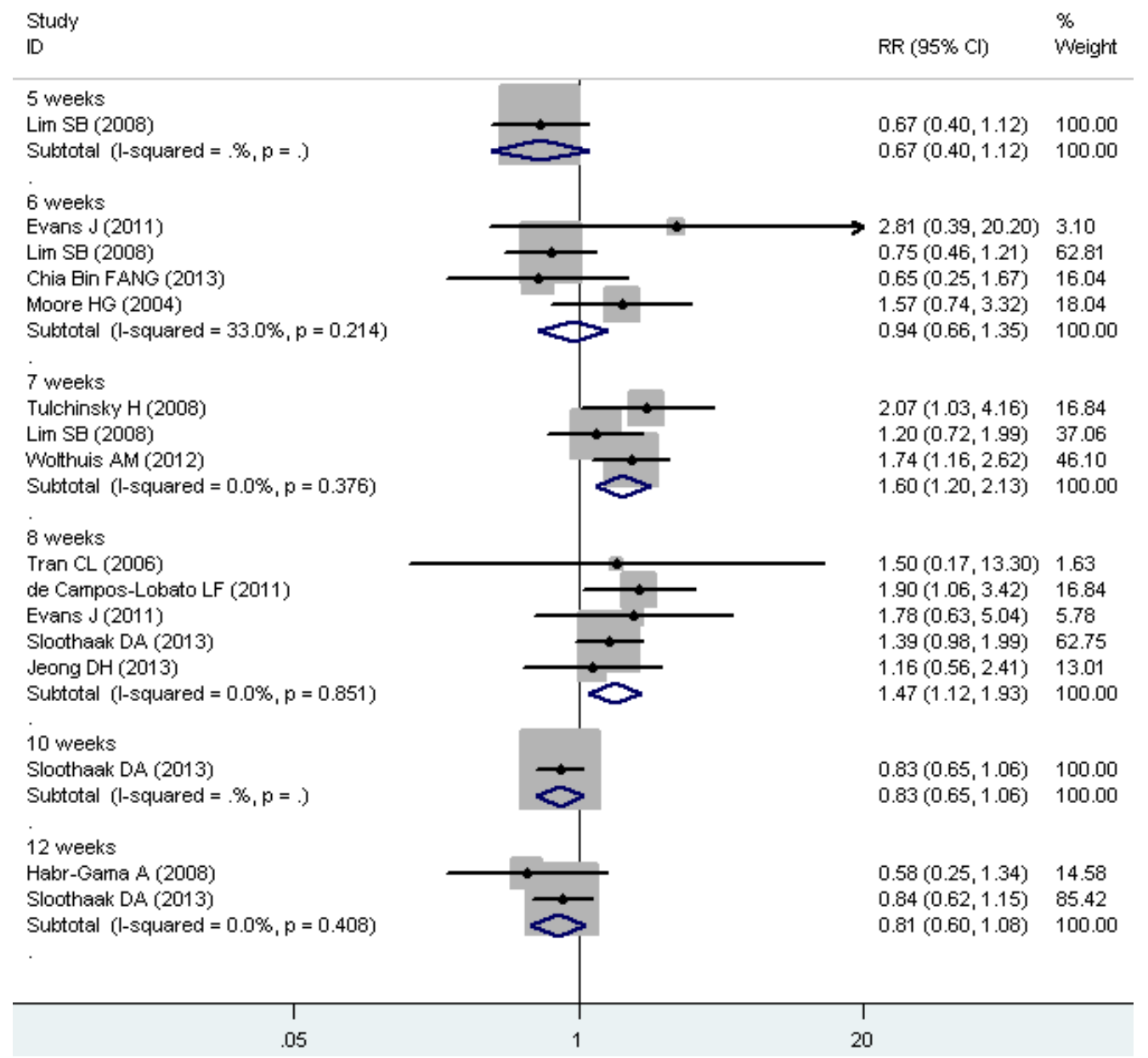

Figure 2: Forest plot

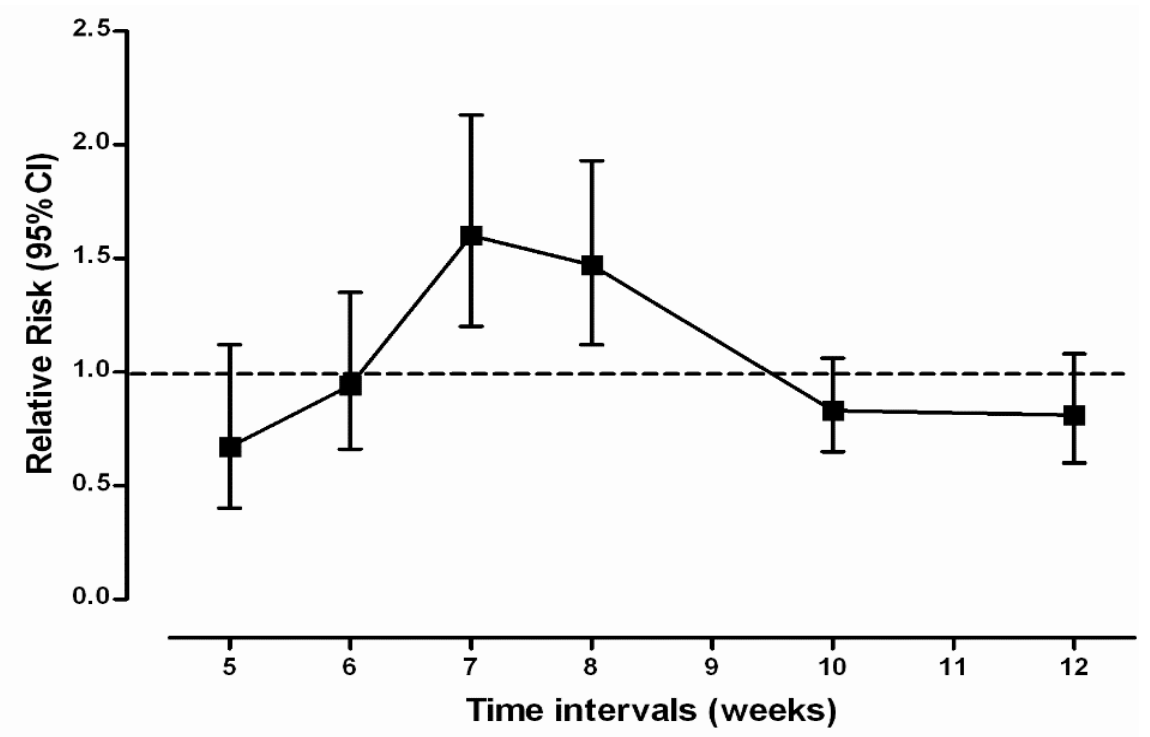

Figure 3: Relationship between time intervals and risk ratio of $\mathrm{pCR}$ rate

of pathologic down-staging. Hence, this time interval has been accepted as the routine practice after CRT for rectal cancer.

The primary tumor down-staging rates were 42.0, 58.0 , and $71.0 \%$ for patients with time intervals of $<6,6-8$, and $>8$ weeks, respectively. An increase in the time interval did not, however, influence the possibility of obtaining a pCR in multivariate analysis [11].

The data in this meta-analysis demonstrate that 
deferring the surgery until the seventh week after CRT leads to the highest possiblity of obtaining $\mathrm{pCR}$ in rectal cancer patients. The $\mathrm{pCR}$ rate was increased by approximate $60 \%$ when patients were operated beyond 7 weeks. Further delay exceeding 10 weeks failed to provide advantages in terms of enhancing the pCR rate. Thus, we establish the optimal cutoff point at 7 weeks to surgically intervene within the established $6-8$ weeks window. A systematic review failed to perform a meta-analysis, due to heterogeneity in the time intervals of included studies. We pooled data from different published time intervals to perform a subgroup analysis.

Our data suggest that no significant difference in pCR rate was found when patients had a waiting interval of $\geq 10$ or 12 weeks. However, a limitation exists in our meta-analysis as only two studies were included in these subgroups. Patients operated on 11 - 13 weeks after CRT had a pCR rate of $25 \%$, compared to $18 \%$ for patients operated on 6 - 8 weeks after CRT [12]. However, increased rate of pCR was not detected when surgery was performed at 10 to 14 weeks in comparison with surgery at 4 to 8 weeks after CRT. Another cohort prospective trial of delaying the interval to 12 weeks between neoadjuvant chemoradiation and surgery is ongoing [13]. However, a few issues need to be addressed. Waiting for a longer period clearly benefits the segment of the patients that achieve a pCR, but over $20 \%$ do not respond to preoperative therapy and, in fact, the primary tumor continues to grow. Another concern of delaying surgery is that longer intervals after preoperative radiation therapy may increase the risk of emergence of distant subclinical tumor, which can grow to a metastasis-yielding volume, and lead to the development of distant metastases [14]. Despite several studies [5,8,10] reporting promising use of imaging technology to help in the monitoring of disease response during preoperative treatment, no robust imaging technology has been established for wide clinical use. Therefore from this viewpoint, further delay of the time interval may be inappropriate. Further studies investigating this issue are needed.

Tumor response towards CRT has been proven to act as an indicator of lower propensity for local or distant recurrence and improved survival. Tumor regression-related indications consist of downsizing, down-staging, complete/almost complete response. As the definitions of downstaging and downsizing vary widely among studies, complete pathological response to neoadjuvant therapy has been recognized as an endpoint in the clinical studies analyzing rectal cancer. In this study, we chose to define pCR when the viable adenocarcinoma cells were absent in surgical sampling, which consisted of lymph node and primary tumors. The summary pCR rate for the entire cohort was $15.8 \%$. There is marked heterogeneity across included studies, probably due to different neo-adjuvant therapy protocols and patients with different stages enrolled in. For patients operated beyond 7 weeks, the sensitivity analyses were performed but excluding, however, the only study in which patients with pCR and near-pCR were included [14].

Many treatment modifications of neo-adjuvant approach have been explored in an effort to increase the percentage of tumor response. These include radiation dose escalation with endo-cavitary boost; induction of chemotherapy during the interval period, use of newer chemotherapeutic agents, and use of regional hyperthermia $[15,16]$. In one reported study [17] exploring the predictors of obtaining pCR, several variables, such as distance between tumor and anal verge, differentiation of pretreated tumors, clinical staging, serum levels of carcinoembryonic antigens, CRT-surgery time interval and irradiation dosage were analyzed by multivariate analysis with $\mathrm{pCR}$ as the dependent variable. Of these variables, an extended interval was the unique vital indicator of obtaining $\mathrm{pCR}$ [18-20].

\section{Limitations of the study}

First, the time intervals after CRT showed significant heterogeneity among the included studies. As surgeons' policy regarding the timing of operation was reported to be the most common factor influencing CRT-surgery intervals by most studies, various potential confounders could have been involved in the choice of interval times for the cases considered. Patients with progressive or stable disease after CRT might have had surgery without further delay after completing CRT. This could have resulted in selection bias. Second, although time intervals of 7 weeks may result in highest chances of achieving pCR in the present study, it is unclear whether this translates into long-term clinical benefit. Third, although all data were extracted from retrospective studies, but the majority of included studies, however, reported on patients with similar baseline demographic and oncologic characteristics in both groups.

\section{CONCLUSION}

The findings of this meta-analysis demonstrate the fact that radical operation carried out after 7 weeks of CRT probably leads to the highest rate 
of pCR. Nevertheless, the correlation between time interval and the long-term survival rate needs to be further elucidated.

\section{DECLARATIONS}

\section{Conflict of Interest}

No conflict of interest associated with this work.

\section{Contribution of Authors}

The authors declare that this work was done by the authors named in this article and all liabilities pertaining to claims relating to the content of this article will be borne by them.

\section{REFERENCES}

1. Scappaticci FA, Fehrenbacher $L$, Cartwright $T$, Hainsworth JD, Heim W, Berlin J, Kabbinavar F, Novotny W, Sarkar S, Hurwitz H. Surgical wound healing complications in metastatic colorectal cancer patients treated with bevacizumab. J Surg Oncol 2005; 91: 173-180.

2. Ranpura V, Hapani S, Wu S. Treatment-related mortality with bevacizumab in cancer patients: a meta-analysis. JAMA 2011; 305:487-494.

3. Van Cutsem E, Tabernero J, Lakomy $R$, Prenen $H$, Prausova J, Macarulla J, Ruff $P$, van Hazel GA, Moiseyenko V, Ferry $D$, et al. Addition of aflibercept to fluorouracil, leucovorin, and irinotecan improves survival in a phase III randomized trial in patients with metastatic colorectal cancer previously treated with an oxaliplatinbased regimen. J Clin Oncol 2012; 30:3499-3506.

4. Douillard J.Y, Siena S, Cassidy J, Tabernero J, Burkes R, Barugel $M$, Humblet $Y$, Bodoky $G$, Cunningham $D$, Jassem J, et al. Randomized, phase III trial of panitumumab with infusional fluorouracil, leucovorin, and oxaliplatin (FOLFOX4) versus FOLFOX4 alone as first-line treatment in patients with previously untreated metastatic colorectal cancer: the PRIME study. J Clin Oncol 2010; 28:4697-4705.

5. Jonker DJ, O'Callaghan CJ, Karapetis CS, Zalcberg JR, Tu D, Au HJ, Berry SR, Krahn M, Price T, Simes RJ, et al. Cetuximab for the treatment of colorectal cancer. $N$ Engl J Med 2007; 357:2040-2048.

6. Hurwitz H., Fehrenbacher L, Novotny W, Cartwright T, Hainsworth J, Heim W, Berlin J, Baron A, Griffing S, Holmgren $F$, et al. Bevacizumab plus irinotecan, fluorouracil, and leucovorin for metastatic colorectal cancer. N Engl J Med 2004; 350:2335-2342.

7. Craven I, Crellin A, Cooper R, Melcher A, Byrne P, Sebag-Montefiore $D$. Preoperative radiotherapy combined with 5 days per week capecitabine chemotherapy in locally advanced rectal cancer. $\mathrm{Br} J$ Cancer 2007; 97: 1333-1337.
8. Chan AK, Wong AO, Jenken DA. Preoperative capecitabine and pelvic radiation in locally advanced rectal cancer-is it equivalent to 5-FU infusion plus leucovorin and radiotherapy? Int. J. Radiat Oncol Biol Phys 2010; 76: 1413-1419.

9. Bazarbashi S, El-Bassiouni M, Abdelsalam M, Soudy $H$, Sanea NA, Jabbar AA, Manji M, Fagih M, Ajarim D. A modern regimen of pre-operative concurrent chemoradiation therapy in locally advanced rectal cancer. J. Surg Oncol 2008; 98: 167-174.

10. Bosset JF, Calais G, Mineur L, Maingon P, RadosevicJelic L, Daban A, Bardet E, Beny A, Briffaux A, Collette $L$. Enhanced tumorocidal effect of chemotherapy with preoperative radiotherapy for rectal cancer: preliminary results-EORTC 22921. J Clin Oncol 2005; 23: 56205627.

11. Gérard JP, Conroy T, Bonnetain F, Bouché $O$, Chapet $O$, Closon-Dejardin MT, Untereiner M, Leduc B, Francois $E$, Maurel J, et al. Preoperative radiotherapy with or without concurrent fluorouracil and leucovorin in T3-4 rectal cancers: results of FFCD 9203. J Clin Oncol 2006; 24: 4620-4625.

12. Collette $L$, Bosset JF, den Dulk $M$, Nguyen F, Mineur $L$, Maingon $P$, Radosevic-Jelic $L$, Piérart $M$, Calais $G$, European Organisation for Research and Treatment of Cancer Radiation Oncology Group. Patients with curative resection of cT3-4 rectal cancer after preoperative radiotherapy or radiochemotherapy: does anybody benefit from adjuvant fluorouracil-based chemotherapy? A trial of the European Organisation for Research and Treatment of Cancer Radiation Oncology Group. J Clin Oncol 2007; 25: 4379-4386.

13. Janjan NA, Crane C, Feig BW, Cleary $K$, Dubrow $R$, Curley $S$, Vauthey JN, Lynch $P$, Ellis $L M$, Wolff $R$, et al. Improved overall survival among responders to preoperative chemoradiation for locally advanced rectal cancer. Am J Clin Oncol 2001; 24: 107-112.

14. Sauer $R$, Becker $H$, Hohenberger W, Rödel $C$, Wittekind $C$, Fietkau R, Martus $P$, Tschmelitsch J, Hager E, Hess $C F$, et al. Preoperative versus postoperative chemoradiotherapy for rectal cancer. $N$ Engl J Med 2004; 351: 1731-1740.

15. Bosset JF, Collette L, Calais G, Mineur L, Maingon $P$, Radosevic-Jelic L, Daban A, Bardet E, Beny A, Ollier $J C$, et al. Chemotherapy with preoperative radiotherapy in rectal cancer. N Engl J Med 2006; 355: 1114-1123.

16. Schrag D, Weiser MR, Goodman KA, Gonen $M$, Hollywood E, Cercek A, Reidy-Lagunes DL, Gollub MJ, Shia J, Guillem JG, Temple LK, et al. Neoadjuvant chemotherapy without routine use of radiation therapy for patients with locally advanced rectal cancer: a pilot trial. J Clin Oncol 2014; 32: 513-518.

17. Torre LA, Bray F, Siegel RL, Ferlay J, Lortet-Tieulent J, Jemal A. Global cancer statistics, 2012. CA Cancer J Clin 2015; 65: 87-108.

18. Xiao J1, Chen Z, Li W, Yang Z, Huang Y, Zheng J, Deng $Y$, Wang $L$, Ren $D$, Peng J, et al. Sandwich-like neoadjuvant therapy with bevacizumab for locally

Trop J Pharm Res, September 2018; 17(9): 1864 
advanced rectal cancer: a phase II trial. Cancer Chemother Pharmacol 2015; 76: 21-27.

19. Resch G, De Vries A, Öfner D, Eisterer W, Rabl $H$, Jagoditsch M, Gnant M, Thaler J; Austrian Breast and Colorectal Cancer Study Group. Preoperative treatment with capecitabine, bevacizumab and radiotherapy for primary locally advanced rectal cancer-a two stage phase II clinical trial. Radiother Oncol 2012; 102: 10-13.
20. Dellas K, Höhler $T$, Reese $T$, Würschmidt $F$, Engel $E$, Rödel C, Wagner W, Richter M, Arnold D, Dunst J. Phase II trial of preoperative radiochemotherapy with concurrent bevacizumab, capecitabine and oxaliplatin in patients with locally advanced rectal cancer. Radiat Oncol 2013; 8: 90. 